\title{
Manifestaciones urológicas de los accidentes vásculo-medulares
}

\author{
Luján Marco S, García Fadrique G, Ramírez Backhaus M, Arlandis Guzmán S, \\ Martínez Agulló E, Jiménez Cruz JF.
}

Unidad de Neurourología. Servicio de Urología. Hospital Universitario La Fe.Valencia.

Actas Urol Esp. 2008;32(9):926-930

\section{RESUMEN}

MANIFESTACIONES UROLÓGICAS DE LOS ACCIDENTES VÁSCULO-MEDULARES

Introducción: Nuestro objetivo es describir las características anatomoclínicas de los accidentes vasculomedulares, así como los métodos diagnósticos y terapéuticos empleados.

Material y Métodos: Estudio retrospectivo desde 1982 hasta 2007, que incluye a 65 pacientes diagnosticados de AVM. Se realizó anamnesis general con exploración neurológica completa y estudio diagnóstico por imagen. La evaluación urodinámica consistió en cistomanometría, estudio de presión flujo y electromiografía del esfínter externo tras la fase de shock medular.

Resultados: De los 65 pacientes (27 mujeres-38 hombres), con una mediana de edad de 63 años (15-87 años), 28 pacientes (43\%) presentaron lesión alta (superior a T7), 32 (49\%)lesión media (entre T7-L2) y 4 (6\%) baja (inferior a L2). El debut clínico más frecuente fue la paraplejía fláccida con retención aguda de orina. La etiología del AVM se determinó mediante pruebas de imagen, principalmente Resonancia Magnética. El estudio urodinámico mostró los siguientes resultados: lesión medular alta con hiperactividad vesical (asociando o no disinergia) 9 pacientes $(47,4 \%)$ y 6 pacientes $(31,5 \%)$ con arreflexia; lesión medular media con hiperactividad 11 pacientes $(47,8 \%)$ y con arreflexia 7 pacientes $(30,4 \%)$; lesión medular baja con hiperactividad un paciente (33\%), arreflexia en otro (33\%).

Conclusiones: No existe una clara correlación entre el nivel anatómico lesionado y el comportamiento vésicoesfinteriano. Esto es debido a que las lesiones medulares de origen vascular suelen ser incompletas y parcheadas, afectándose diferentes niveles. Es necesario, por tanto, realizar una exhaustiva exploración neurológica y urológica en estos pacientes, para poder establecer el patrón funcional que nos permita indicar el tratamiento idóneo.

Palabras clave: Urodinámica. Accidente vásculo-medular.

\section{ABSTRACT \\ UROLOGICAL FINDINGS IN SPINAL CORD ISCHEMIA}

Introduction: Spinal cord ischemia is a circulatory disorder of acute or subacute establishment and neurological clinic of medullar section, usually incomplete. We describe clinical and anatomical characteristics, as well as diagnostic methods and therapeuthic used in these patients.

Material and methods: since 1987 to 2007, 65 patients were diagnosed of spinal cord ischemia in our hospital. Clinical interview, neurological examination and image study were performed. Urodynamic study was performed after medullar shock phase, including cystometry, pressure flow study and external urinary sphincter electromyography. We have reviewed treatment applied in these patients.

Results: 65 patients (27 women and 38 men), median age 63 years (15-87). 28 patients (43\%) presented high lesion (upper to T7), 32 (49\%) middle (T7-L2) and 4 (6\%) low lesion (below L2). Flaccid paraplegia and acute urinary retention were the most frequent clinical findings at the begining. Aetiology was determined mainly by Magnetic Resonance. Urodynamic study revealed: 9 patients $(47,4 \%)$ with high spinal cord lesion and detrusor overactivity (with or without dyssynergia), and $6(31,5 \%)$ with arreflexia; 11 patients $(47,8 \%)$ with middle spinal cord lesion and detrusor overactivity and 7 patients with arreflexia $(30,4 \%)$; one patient with low spinal cord lesion and detrusor overactivity, and another one with arreflexia (33\%).

Conclusions: there is a good correlation among lesion level and clinical findings in traumatic spinal cord injuries, but this is not the same in spinal cord ischemia. Probably, incomplete and patched lesions in vascular spinal cord injuries could explain this lack of correlation. Therefore, it is mandatory to performe an exhaustive neurological and urological evaluation of these patients in order to select the best treatment and prevent upper urinary tract damage in the future.

Keywords:Urodynamic. Spinal cord ischemia. 
$\mathrm{E}$ accidente vasculomedular (AVM) consiste en un trastorno circulatorio de instauración aguda o subaguda y una clínica neurológica de sección medular, habitualmente incompleta. Se trata de una patología poco frecuente, con pocas series publicadas en la literatura ${ }^{1-3}$. La incidencia de lesionados medulares en España fue valorada en 505 casos anuales para una población de 38.500.000 habitantes, siendo la incidencia de 0,8 por cada 100.000 habitantes para las lesiones traumáticas y de 0,5 en las no traumáticas ${ }^{4}$. Dentro de las causas médicas, las lesiones de origen vascular ocupan el segundo lugar seguido de los tumores ${ }^{5}$.

Las características de la disfunción vesicoesfinteriana en el lesionado medular pueden ser variables, desde una mínima disfunción, apenas perceptible, hasta una alteración completa y dependerán en parte de la especial distribución anatómica de la vascularización medular. El lesionado medular, al igual que el paciente con mielomeningocele, suele tener al urólogo como al médico que con mayor frecuencia consulta a lo largo de su vida. Nuestro objetivo será por tanto conocer la clínica y los hallazgos urodinámicos que presentan estos pacientes con el fin de obtener un manejo óptimo respecto a la sintomatología del tracto urinario inferior secundario a la lesión medular que facilite el correcto vaciamiento vesical y evite el deterioro del tracto urinario superior.

\section{MATERIAL Y MÉTODOS}

Efectuamos un estudio descriptivo retrospectivo, incluyendo a 65 pacientes diagnosticados de accidente vasculomedular (AVM) en nuestro centro (27 mujeres y 38 hombres) en los últimos 25 años. Las edades de la muestra oscilaron entre 15 y 87 años con una mediana de 63 años. El diagnóstico se realizó en un primer momento mediante exploración física y pruebas de imagen. Cabe destacar que en la mayoría se utilizó la Resonancia Magnética (36 casos), y en el resto Tomografía Computerizada o arteriografía. Posteriormente, y pasada la fase de shock medular se sometió a los pacientes a una exploración neurológica y urológica, así como a estudio urodinámico, que incluyó cistomanometría y estudio presiónflujo entre los 3 y 8 meses transcurrido el episodio agudo.

\section{RESULTADOS}

Las causas de los accidentes vasculo-medulares identificadas en nuestra serie las agrupamos en tres grupos: mecánicas, vasculares y de origen iatrogénico. En 12 pacientes no se logró identificar la causa.

- Causas Mecánicas: 7 pacientes $(13,4 \%)$, que incluyen latigazo cervical (2), fractura vertebral (2), escoliosis severa (1) y ejercicio físico intenso o no habitual (2).

- Causas Vasculares: 44 pacientes $(63,5 \%)$; postcirugía raquídea (1), postraquianestesia (2), postcirugía cardiaca (1), postcirugía vascular (4), hipotensión mantenida (3), rotura aórtica (2), infarto agudo de miocardio (1), postangioplastia (1), malformaciones arteriovenosas (11), hemangioblastoma (2), ependimoma (1), aterosclerosis (5), hematoma subdural (5), hematoma epidural (2), hematoma intradural (1), trombosis arterial (2).

- Yatrógenas: 2 pacientes (3,8 \%); tras embolización de arterias bronquiales para el tratamiento de hemoptisis severa.

Respecto al nivel lesional hemos dividido a los pacientes en tres grupos, en función de la localización vertebral de la lesión. Presentaron lesión a nivel medio (comprendida entre T7-L2) 33 pacientes (49\%), 28 pacientes (43\%) lesión alta (por encima de T7) y sólo en 4 pacientes (6\%) la lesión era baja (por debajo de L2). Respecto a la clínica neurológica de sección medular la mayoría presentaban lesiones incompletas; 52 pacientes $(81,2 \%)$ frente a 13 pacientes $(18,7 \%)$ en los que la lesión medular fue completa.

La manifestación urológica más frecuente fue la retención aguda de orina, en 45 pacientes (69\%), seguido de síntomas de tracto urinario inferior como dificultad para iniciar la micción, chorro débil, sensación de vaciado incompleto asociado y/o diferentes grados de urgencia miccional con o sin incontinencia que presentaron 13 pacientes (20\%), mientras que 7 pacientes (11\%) no presentaron ningún tipo de sintomatología de tipo urológico. Con respecto a la clínica neurológica, la mayoría de los pacientes presentaron paraparesia fláccida (23 pacientes, 35,9\%), mientras que el resto de pacientes debutaron con diversos grados de paresia, asociando algunos de ellos dolor en raquis.

Efectuamos estudio urodinámico a 45 pacientes. El principal hallazgo fue hiperactividad del detrusor, con o sin disinergia. De este modo, 9 pacientes $(47,4 \%)$ con 
lesión alta, 11 (47,8 \%) con lesión media y 1 paciente $(33 \%)$ con lesión baja presentaron este hallazgo urodinámico. Cabe destacar que 6 pacientes $(31,5 \%)$ con lesión medular alta presentaron arreflexia mientras que sólo un paciente (33\%) de los pacientes con lesiones bajas se encontró este hallazgo. En Tabla 1 se resumen los hallazgos urodinámicos.

En el momento agudo de presentación del AVM, tan sólo 5 pacientes $(7,6 \%)$ precisaron un procedimiento quirúrgico urgente, siendo el resto manejados con tratamiento médico.

El tratamiento urológico seguido por estos pacientes tras el accidente vasculo medular fue: en 18 pacientes (40\%) autocateterismo limpio intermitente, asociándolo a $\alpha$-bloqueantes en 5 pacientes y a anticolinérgicos en un paciente; 4 pacientes $(8,8 \%)$ recibieron un $\alpha$-bloqueantes, 15 pacientes (33,3\%) anticolinérgicos, mientras que un paciente $(2,2 \%)$ necesitó la asociación de anticolinérgico y $\alpha$-bloqueante; 4 pacientes $(8,8 \%)$ no requirieron tratamiento de ningún tipo.

\section{DISCUSIÓN}

La micción es un proceso fisiológico complejo que requiere la integración de diversas estructuras. Córtex cerebral, diversos centros encefálicos, núcleos del troncoencéfalo y de la médula espinal lumbosacra han de coordinarse para lograr un correcto vaciado vesical ${ }^{6}$.

Cuando se produce una lesión medular en cualquier localización tiene lugar una primera fase de shock caracterizado funcional y urodinámicamente por una arreflexia del detrusor y una hipoactividad del esfínter uretral externo, con posibilidad de aparición de resistencia uretral por actividad del músculo liso del cuello vesical. Gran parte de las manifestaciones clínicas que observamos en esta fase sufrirán una modificación a lo largo del tiempo, de modo que superada la fase de shock medular podremos establecer el patrón funcional con el fin de determinar el tratamiento ${ }^{7}$.
Cuando las lesiones son completas y a un determinado nivel, es fácil predecir la repercusión que tendrá sobre la dinámica miccional. Así ocurre, por ejemplo, en las lesiones medulares de tipo traumático. En cambio, en los AVM las lesiones suelen ser incompletas, como hemos recogido en nuestra serie, $\mathrm{y}$ afectan a distintos niveles, siendo por tanto menos previsible el trastorno funcional subyacente.

En lo que se refiere a la vascularización de la médula raquídea existen una serie de singularidades que dificultan el estudio de sus lesiones. Adamkievicz, en 1882, describió la vascularización medular; desde entonces se ha avanzado mucho en este campo, gracias a las nuevas tecnologías y pruebas de imagen. Sin embargo sigue siendo difícil correlacionar el aspecto puramente anatómico con la clínica de cada paciente con lesión vasculo medular, así como predecir las secuelas de la misma ${ }^{8}$.

A nivel cervicodorsal la médula espinal recibe irrigación a partir de la vía espinal anterior, formada por la fusión de ramas radiculomedulares de las arterias vertebrales. A nivel dorsal medio la arteria radiculomedular anterior es una arteria única y fina. A nivel dorsolumbar la arteria de Adamkievicz nutre la cara anterior de la médula y tiene anastomosis con las arterias radiculares posteriores.

Por tanto la región cervical y dorsolumbar tienen conexiones que pueden suplir el aporte sanguíneo en el territorio anterior, en caso de oclusión de una arteria radiculomedular. Sin embargo, a nivel dorsal medio la vía anterior está formada exclusivamente por una arteria única y de pequeño calibre, de manera que una lesión que ocluya toda su luz producirá una isquemia que no podrá ser suplida por circulación colateral, de modo que la repercusión clínica será mayor ${ }^{9}$

En nuestro estudio hemos incluido todos aquellos pacientes que presentan manifestaciones neurológicas compatibles con lesión medular, y cuyo origen es vascular. La mayor parte de los casos tienen el origen de la lesión en un problema inicial-

Tabla 1: Resultados del estudio urodinámico en función del nivel de la lesión medular

\begin{tabular}{|c|c|c|c|c|c|}
\hline & $\begin{array}{c}\text { Hiperactividad }+/- \\
\text { Disinergia } \\
\mathbf{n}(\%)\end{array}$ & $\begin{array}{c}\text { Arreflexia } \\
\mathbf{n}(\%)\end{array}$ & $\begin{array}{c}\text { Hipoactividad } \\
\mathbf{n}(\%)\end{array}$ & $\begin{array}{c}\text { Hipoacomodación } \\
\mathbf{n}(\%)\end{array}$ & $\begin{array}{c}\text { Normal } \\
\mathbf{n}(\%)\end{array}$ \\
\hline Alta & $9(47,4)$ & $6(31,5)$ & $1(5,26)$ & 0 & $3(15,78)$ \\
\hline Media & $11(47,8)$ & $7(30,4)$ & 0 & $1(4,3)$ & $4(17,3)$ \\
\hline Baja & $1(33,3)$ & $1(33,3)$ & 0 & 0 & $1(33,3)$ \\
\hline
\end{tabular}


mente vascular, como malformaciones arteriovenosas $^{10-12}$ o hipotensión mantenida (en relación con shock hipovolémicos o sépticos). Cabe destacar que hemos recogido numerosos casos en los que el AVM aparece en el postoperatorio de diferentes intervenciones sobre la columna vertebral o el aparato cardiovascular tal y como se recogen en diferentes casos clínicos publicados ${ }^{13,14}$. Lejos ha quedado la época en la que la sífilis constituía una causa frecuente de isquemia medular.

Respecto a la clínica que aparece tras producirse el accidente vascular medular a nivel cervical, esta cursa con intensa cefalea o dolor cervical, pudiendo asociar también un episodio isquémico cerebral $^{15}$. En nuestra experiencia, sin embargo, sólo 11 pacientes debutaron con dolor a nivel de raquis.

Es bien conocida la fisiopatología de la dinámica miccional, sabiendo que, por ejemplo, las lesiones por encima del núcleo parasimpático sacro, al desconectar al mismo del núcleo pontino cursan con hiperactividad del detrusor, así como una hiperactividad uretral, generando disinérgica vesicoesfinteriana y produciendo altas presiones intravesicales ${ }^{7}$. Sin embargo en nuestra serie hemos observado que sólo el 47,4 $\%$ de los pacientes con lesiones altas presentaban hiperactividad del detrusor, y un 31,5\% manifestaron arreflexia en el estudio urodinámico.

Del mismo modo, sabemos que cuando se produce una lesión medular baja (sacras) la lesión se localiza sobre los núcleos medulares de la micción aparece un detrusor hipoactivo o arrefléxico, junto con una hipoactividad del esfínter externo, debido a la destrucción del parasimpático sacro. La vejiga se vacía cuando está llena, sin contracciones del detrusor, al no existir ninguna resistencia uretral que se oponga a la salida de orina. Sin embargo, hemos encontrado que un 33\% de las lesiones bajas presentaron hiperactividad del detrusor.

Por lo tanto, encontramos una falta de correlación anatomoclínica, dado que en los AVM el daño no se produce a un nivel metamérico exclusivo (como ocurre en los traumatismos), sino que se trata de lesiones difusas y de mayor extensión ${ }^{2,15,16}$.

Esto nos conduce a que la selección de un tipo u otro de tratamiento no dependerá tanto de la localización anatómica de la lesión, como de los hallazgos urodinámicos. Los objetivos fundamentales del tratamiento de la vejiga neurógena secundaria a lesión medular serán tratar la hiperactividad vesical y disminuir la resistencia uretral, con el fin de conseguir un llenado a bajas presiones y un vaciado eficaz. Los fármacos más utilizados en estos pacientes han sido los anticolinérgicos y los bloqueantes alfa. Con los primeros tratamos estabilizar el comportamiento vesical para evitar la incontinencia y mejorar la acomodación; con los segundos disminuiremos la resistencia uretral y la presión del detrusor de fuga con el fin de facilitar el vaciado. Cerca de la mitad de los pacientes iniciaron tratamiento con cateterismo limpio intermitente asociado o no a fármacos con el fin de liberar al paciente del sondaje uretral permanente, vaciar la vejiga para prevenir la sobredistensión, la incontinencia y evitar las complicaciones derivadas de éstas.

El pronóstico funcional (tanto a nivel motor como de función vesicouretral), de estos pacientes superada la fase de shock medular es desconocido. De Seze et al. ${ }^{8}$ analizaron a 23 pacientes con isquemia medular, evaluándose los hallazgos urodinámicos y clínicos según los criterios de la Asociación Americana de Lesionados Medulares (ASIA-IMSOP). Se evidenció que los pacientes cuya lesión vascular no alcanzaba el cono medular presentaban mejores resultados funcionales.

El paciente que ha sufrido una mielopatía vascular se beneficiará, por tanto, de una exploración neurourológica completa, así como de un estudio urodinámico exhaustivo, con el fin de establecer un diagnóstico correcto ${ }^{17,18}$. De todo ello derivará la selección terapéutica idónea para conseguir un vaciado vesical completo a bajas presiones, que nos permita la preservación del tracto urinario superior y mantener la continencia ${ }^{8,19}$.

\section{REFERENCIAS}

1. Gomelsky A, Lemack GE, Weld KJ, Dmochowski RR. Urodynamic patterns following isquemic spinal cord events. J Urol. 2003;170(1):122-125.

2. Batista JE, Bauer SB, Shefner JM, Kelly MD, Darbey MD, Siroky MB. Urodynamic findings in children with spinal cord ischemia. J Urol. 1995; 154(3):1183-7.

3. Benedicto Redón A, Soria Lafuente M , Budía Alba A, Burgués Gassión JP , Martínez Agulló E. Alteraciones urodinámicas en el accidente vascular medular .Urod A. 1998,11 (4):147-151.

4. Garcia Reneses J , Herruzco Cabrera R, Martínez Moreno M. Epidemiological study of spinal cord injury in Spain 1984-1985. Paraplegia. 1991; 28: 180.

5. Victoria Iñigo E , Martínez Agulló E , Crisponi I , Mañez A , Cuñat E, Martínez R. Complicaciones del tracto urinario superior durante el primer año de evolución de una lesión medular. Urod A. 1990; 2: 100. 
6. Martínez Agulló E. et al. Dinámica de la micción y vejiga neurógena. En Martínez Agulló E, Ruiz Cerdá JL, Arlandis Guzmán S , Gómez Pérez L. Disfunción vesical en el enfermo neurológico. Prous Science, Barcelona 2003;17-18:32-34.

7. Arlandis Guzmán S , Martínez Agulló E, Jiménez Cruz J.F. Clasificación y diagnóstico de la vejiga neurógena. En Jiménez Cruz J.F, Rioja Sanz L.A. Tratado de Urología. $2^{\circ}$ Edición. Prous Science. 2006:1071-1074.

8. De Seze M, de Seze M, Joseph PA, Wiart L, Nguyen PV, Barat M. Functional prognosis of paraplegia due to cord ischemia: a retrospective study of 23 patient. Rev Neurol (Paris). 2003; 159(11):1038-1045

9. Silver JR, Buxton PH. Spinal stroke. Brain. 1974;97(3):539550.

10. Symon, L; Kuyama, H; Kendall B. Dural arteriovenous malformartions of the spine. Clinical features and surgical results in 55 cases. J Neurosurg. 1984;60(2):238-247.

11. Rosenblum, B; Oldfield, E.H. Spinal sarteriovenous malformations: a comparison of dural arteriovenous fistulas and intradural AVM in 81 patients. J Neururg.1987;67(6):795-802.

12. Rojas del Castillo CJ, Verdú Más F, Alfonso Diago L, Miró Vila R. Mielopatía por malformación arteriovenosa de la médula espinal: presentación de tres casos. Rehabilitación. 1997; 31(4):311314.

13. Beauchesne LM, Mailis A, Webb GD. Neuropathic pain syndrome as an occult manifestation of injury of the spinal cord after surgical repair of aortic coarctation. Cardiol Young. 2000; 10(4):413-415.
14. Cook, A.W. Oclussion of the abdominal aorta and dysfunction of the spinal cord, a clinical syndrome. Bull NY Acad Med. 35: 479-489.

15. Arnold M, Bousser MG Clinical manifestations of vertebral artery dissection. Front Neurol Neurosci. 2005;20:77-86.

16. Kaplan, S.A , Chancellor, M.B. Bladder and sphinter behaviour in patients with spinal cord lesions. J Urol.1991;146:(1)113117.

17. Kotkin L, Milam DF. Evaluation and management of the urologic consequences of neurologic disease. Tech Urol. 1996; 2(4): 210-219.

18. Nitti VW, Adler H, Combs A.J. The role of urodynamics in the evaluation of voiding dysfunction in men after cerebrovascular accident. J Urol. 1996;155(1):263-266.

19. Yokoyama T, Yoshimura N, Dhir R, Qu Z, Fraser MO, Kumon $\mathrm{H}$, de Groat WC, Huard J, Chancellor MB. Morphological and Functional Factors Predicting Bladder Deterioration After Spinal CordInjury. J Urol. 1996;155(1):271-274.

Correspondencia autor: Dr. S. Luján Marco

Unidad de Neurourología. Servicio de Urología.

Hospital Universitario La Fe

Avda. Campanar, 21 - 46009 Valencia

Tel.: 963862700

E-mail autor: slujanmarco@comv.es

Información artículo: Original - Vejiga neurógena

Trabajo recibido: abril 2008

Trabajo aceptado: mayo 2008 